\title{
Depression in Patients with Diabetes: Does It Impact Clinical Goals?
}

\author{
William A. Rush, PhD, Robin R. Whitebird, PhD, Monica R. Rush, BS, \\ Leif I. Solberg, MD, and Patrick J. O'Connor, $M D, M P H$
}

Introduction: To examine whether depressive symptoms are associated with achievement of recommended goals for control of glucose, lipids, and blood pressure among patients with diabetes.

Methods: We used a prospective cohort study of 1223 adults with diabetes that obtained self-reported depression symptoms from a survey. Medication use was obtained from claims data, and pharmacy and clinical data were obtained by manual review of paper medical records.

Results: Diabetes patients with depression symptoms were less likely to be at their glucose goal (43\% vs $50 \% ; P=.0176)$ but more likely to be at their blood pressure goal $(57 \%$ vs $51 \% ; P=.0435)$. The association between lipids and depression symptoms was related to a lower rate for low-density lipoprotein testing $(56 \%$ vs $68 \% ; P<.0001)$. Treatment with antidepressants resulted in a greater percentage achieving glucose and blood pressure goals but not lipid goals.

Conclusions: Depression seems to have a variable impact on achieving these clinical goals, perhaps because the goals have differing measurement logistics and biological profiles. Further research is needed to learn whether better treatment of depressive symptoms leads to improvements in meeting diabetes clinical goals. (J Am Board Fam Med 2008;21:392-397.)

Patients with diabetes have nearly twice the risk of comorbid depression as the general population. In a recent study of the epidemiology of major depressive disorder (MDD), from the National Comorbidity Survey Replication, the lifetime prevalence of depression in the general population was estimated at $16.2 \% .{ }^{1} \mathrm{~A}$ recent meta-analysis of the prevalence of comorbid depression in diabetes patients estimated their lifetime prevalence at $28.5 \% .^{2}$ Depression has also been found to be linked to increased cardiovascular risk, ${ }^{3-5}$ one of the primary causes of mortality among diabetes patients.

A number of studies have investigated the impact of depression on glucose control ${ }^{6-13}$ in diabetes patients. These studies are divided, with some finding a relationship between the presence of depression and glucose control and others finding no

This article was externally peer reviewed.

Submitted 16 April 2007; revised 7 May 2008; accepted 12 May 2008.

From the HealthPartners Research Foundation, Minneapolis, Minnesota.

Funding: This project was funded by a grant from the Agency for Healthcare Research and Quality, number R01HS09946-01A1.

Conflict of interest: none declared.

Corresponding author: William A. Rush, $\mathrm{PhD}$, HealthPartners Research Foundation, PO Box 1524, MS 21111R, Minneapolis, MN 55440-1524 (E-mail: Bill.A.Rush@healthpartners.com). indication of any association. ${ }^{7,9}$ Few studies have assessed depression's impact on the equally important control of blood pressure or lipids. ${ }^{13}$ Control of these frequently associated risk factors is an important and cost-effective strategy to reduce major diabetes complications such as heart attacks, strokes, nephropathy, and retinopathy. ${ }^{14,15}$

This study is a secondary analysis of data from Project QUEST, a prospective cohort study that investigated the relationship of medical group, clinic, physician, and patient characteristics on best care practices for diabetes care. The analysis time periods consist of two 1-year intervals on either side of the date of receipt of a patient survey in 2001. We tested the hypothesis that reported depressive symptoms would be associated with a reduced ability to reach recommended goals for control of glucose, lipid, and blood pressure.

\section{Methods}

Eligible study subjects were adults (age 18 and older) who were insured by HealthPartners (HP) health plan, had received care at a HP-contracted medical group in Minnesota, had diagnosed diabetes, and were randomly selected for participation in Project QUEST. Of those identified, 4942 were sent a survey, which was completed and returned by 3838 (78\%). Of these, 2121 (55\%) gave permission 
for review of their medical record. Because of the need to use antidepressant medication as a proxy for depression treatment, we excluded the 898 without pharmacy coverage, leaving 1223 for this analysis.

Adults were classified as having diabetes if they had either (1) 1 or more inpatient or 2 or more outpatient ICD9 diagnosis codes for diabetes mellitus (250.xx) within a defined 1-year period or (2) had a filled prescription for a diabetes-specific medication within the same defined 1 -year period. ${ }^{16}$ This method of diabetes identification has been validated and has estimated sensitivity of 0.91 , positive predictive value of 0.94 , and specificity of 0.99 .

Self-reported depression-related symptoms were identified from the survey, which asked 2 questions from the PHQ-2 screening tool for depression:

1. During the past month have you been bothered by feeling down, depressed, or hopeless?

2. During the past month, have you often been bothered by little interest or pleasure in doing things?

These 2 questions have been shown to have a high sensitivity of between $89 \%$ and $97 \%$, with a more variable specificity (51\% to $91 \%$ ) for depression case-finding. ${ }^{17,18}$ We stratified respondents by those who had no positive responses to these questions versus those with one or more positive responses to the questions to create the independent variable of reported depression symptoms.

Dependent variables included levels of glycohemoglobin (A1c), low-density lipoprotein (LDL), systolic blood pressure (SBP), and diastolic blood pressure (DBP). These data were obtained by manual review of paper medical records and were collected for the 1-year period after the survey. These measures were classified in 2 ways:

1. Was a measurement available within the study year, and

2. Was the patient at the desired goal for the particular clinical measure (A1c $\leq 7.0 ; \mathrm{LDL} \leq$ $100 ; \mathrm{SBP} \leq 130 ; \mathrm{DBP} \leq 80)$ in each defined year.

No data were collected from the medical record review relative to depression status.
Bivariate comparisons were performed using $t$ tests and $\chi^{2}$ statistics, as appropriate. When multiple measures were available for a patient, the last one in the 1 -year period was used in analysis. Coding of achievement of clinical goals and the presence of symptoms for depression were coded as bivariate variables. Age and gender were controlled for as of the survey date. All analyses were conducted using SAS 9.1 (SAS Institute Inc., Cary, NC).

Treatment with an antidepressant medication was determined by the presence of a claim for a pharmacy fill during the period of interest for a medication with a GPI group code of 58 (ie, antidepressants). Although there was no way to know whether the fill was for the treatment of depression or one of the other uses of these medications, because the subject was exhibiting symptoms of depression it seems likely that in most cases this was the intended use of the antidepressant.

\section{Results}

A comparison of the demographics of the patients responding to the survey with the subset analyzed in this study found that among the study subjects in this substudy, more were never married $(7.1 \%$ vs $3.8 \%$ ), fully employed ( $38.7 \%$ vs $28.9 \%$ ), had a lower income (18.6\% vs $25.1 \%)$, and had a slightly higher body mass index (31.0 vs 29.2). There were no differences in educational level, race, or Hispanic ethnic status.

Of the 1223 subjects, 471 (38.5\%) reported some symptoms of depression during the past month. Table 1 contains a comparison of demographic data for the study population reporting no depression symptoms versus those indicating at least 1 depression question positively.

Among the patients reporting symptoms of depression, $36 \%$ had a diagnosis of depression in the year before the survey and $26 \%$ had a depression diagnosis in the year after the survey. Of the patients with symptoms of depression on the survey, the percentage receiving medication treatment increased from $41 \%$ to $47 \%$ between the year before and after the survey.

There was no relationship between a report of depression symptoms and A1c testing rates, but there was a pronounced relationship to being at A1c goal (Table 2). Those with depression symptoms were less likely to be at goal ( $43 \%$ vs $50 \%$; $P=.0176)$. Among the subset of patients who were 
Table 1. Comparison of Sociodemographic Factors in Those With Diabetes With and Without Depressive Symptoms

\begin{tabular}{|c|c|c|}
\hline Variable & $\begin{array}{l}\text { Diabetes Patients with No Depressive Symptoms } \\
\qquad(\mathrm{n}=752)\end{array}$ & $\begin{array}{l}\text { Diabetes Patients with Depressive Symptoms } \\
\qquad(\mathrm{n}=471)\end{array}$ \\
\hline \multicolumn{3}{|l|}{$\operatorname{Sex}(\%[n])$} \\
\hline Male & $57.7(434)$ & $52.9(249)$ \\
\hline Female & $42.3(318)$ & $47.1(222)$ \\
\hline Age (years) & 61.2 & 58.7 \\
\hline \multicolumn{3}{|l|}{ Race $(\%[\mathrm{n}])$} \\
\hline White & $95.1(708)$ & $90.1(419)$ \\
\hline Black & $1.5(11)$ & $5.0(23)$ \\
\hline Asian & $1.2(9)$ & $2.4(11)$ \\
\hline Other & $2.3(17)$ & $2.6(12)$ \\
\hline \multicolumn{3}{|l|}{ Marital status (\% [n]) } \\
\hline Married & $73.5(540)$ & $66.2(303)$ \\
\hline Single & $7.1(52)$ & $9.0(41)$ \\
\hline Separated/divorced & $8.0(59)$ & $12.7(58)$ \\
\hline Widowed & $9.5(70)$ & $12.2(56)$ \\
\hline \multicolumn{3}{|l|}{ Education (\% [n]) } \\
\hline Less than high school & $4.9(36)$ & $7.1(32)$ \\
\hline High school & $28.1(205)$ & $31.5(143)$ \\
\hline Vocational/business & $12.7(93)$ & $13.7(62)$ \\
\hline Some college & $23.6(172)$ & $24.9(113)$ \\
\hline College degree & $16.3(119)$ & $15.2(69)$ \\
\hline Graduate school & $14.4(105)$ & $7.7(35)$ \\
\hline \multicolumn{3}{|l|}{ Employment (\% [n]) } \\
\hline Full time & $43.2(317)$ & $44.6(204)$ \\
\hline Part time & $6.8(50)$ & $3.9(18)$ \\
\hline Unemployed & $1.2(9)$ & $5.3(24)$ \\
\hline Retired & $44.3(325)$ & $42.2(193)$ \\
\hline Other & $4.5(33)$ & $3.9(18)$ \\
\hline \multicolumn{3}{|c|}{ Household Income (\% [n]) } \\
\hline$<\$ 25,000$ & $14.0(100)$ & $20.5(93)$ \\
\hline$\$ 25,000$ to $\$ 49,999$ & $31.5(225)$ & $35.1(159)$ \\
\hline$\$ 50,000$ to $\$ 74,999$ & $20.6(147)$ & $18.1(82)$ \\
\hline$\geq \$ 75,000$ & $19.7(141)$ & $16.1(73)$ \\
\hline Won't Answer & $14.3(102)$ & $10.2(46)$ \\
\hline
\end{tabular}

not at goal, those receiving postsurvey antidepressant medication treatment were more likely to achieve their glucose goal in the postsurvey year (48\% vs $38 \% ; P=.0517$; Table 3 ).
LDL levels were associated with reduced testing frequency ( $56 \%$ vs $68 \%$ ), but there were no differences in goal achievement (Table 2). Those treated with antidepressants were no more likely to have

Table 2. Relationship of Presence of Depression to Clinical Outcome Testing Rates and Goal Status in Postsurvey Year

Postsurvey Year Clinical Measures

\begin{tabular}{lccccccc} 
Depression Symptoms & A1c Tested & A1c at Goal & LDL Tested & LDL at Goal & SBP/DBP Tested & SBP at Goal & DBP at Goal \\
\hline No (\%) & 84 & 50 & 68 & 56 & 90 & 51 & 41 \\
Yes (\%) & 81 & 43 & 56 & 49 & 89 & 57 & 45 \\
$P$ & .241 & .0176 & $<.0001$ & .0669 & .549 & .0435 & .1557 \\
\hline
\end{tabular}

A1c, glycohemoglobin; LDL, low-density lipoprotein; SBP, systolic blood pressure; DBP, diastolic blood pressure. 
Table 3. Relationship of Presence of Antidepressant Treatment in Sample with Depression to Clinical Outcome Testing Rates and Goal Status in Postsurvey Year

\begin{tabular}{|c|c|c|c|c|c|c|c|}
\hline \multirow{2}{*}{$\begin{array}{l}\text { Depression Symptoms } \\
\text { on Medication }\end{array}$} & \multicolumn{7}{|c|}{ Postsurvey Year Clinical Measures } \\
\hline & Alc Tested & Alc at Goal & LDL Tested & LDL at Goal & SBP/DBP Tested & SBP at Goal & $\mathrm{DBP}$ at Goal \\
\hline No (\%) & 81 & 38 & 57 & 51 & 85 & 51 & 40 \\
\hline Yes $(\%)$ & 82 & 48 & 55 & 47 & 93 & 64 & 51 \\
\hline$P$ & .7936 & .0517 & .8071 & .5186 & .0075 & .0096 & .0232 \\
\hline
\end{tabular}

A1c, glycohemoglobin; LDL, low-density lipoprotein; SBP, systolic blood pressure; DBP, diastolic blood pressure.

either LDL testing rates or goal achievement (Table 3).

In contrast, patients with depressive symptoms were just as likely to have blood pressure testing (89\% vs $90 \%)$ and more likely to have SBP $(57 \%$ vs $51 \% ; P=.0435)$ but not DBP ( $45 \%$ vs $41 \% ; P=$ $0.1557)$ at goal. Those patients with depression symptoms who subsequently were on antidepressants were even more likely to be at SBP goal (64\% vs $51 \% ; P=.0096$; Table 3 ).

\section{Discussion}

The presence and treatment of depression symptoms seems to have a varying impact on a patient's ability to reach glucose, lipid, and blood pressure clinical goals. Diabetes patients with depression symptoms are less likely to achieve glucose goals, but this improves when they are treated with antidepressants. The achievement of lipid goals does not seem to be affected by the presence of depression symptoms or their treatment, although testing rates are lower in patients with depression symptoms. Only the systolic component of blood pressure was in better control in the population with depression symptoms, and this seems to improve further with treatment with antidepressants.

Why should these 3 measures behave so differently in relation to the presence of depression symptoms and treatment? One possibility is that they have differing measurement logistics and biological profiles that may impact a patient's ability to reach important clinical goals. The glycohemoglobin (A1c) test can be taken at the clinic lab without any prior preparation and in association with any clinic visit; it is an average of the level of glucose in the blood over the prior approximately 3 months. In contrast, blood pressure is generally taken as the patient is waiting to see the physician and it is a point measurement in time with considerable variation. Taking the fasting lipid test is logistically complex, because the patient is required to fast for at least 12 hours and then travel to a blood draw site. Biologically, it represents measurements of the body's natural lipid levels, largely independent of dietary intake.

Because A1c testing rates were high in this population $(>85 \%)$ and a patient only had to have a single test within a study year to be classified as tested, it is not surprising that there was no significant difference in A1c testing rates. Because A1c is a measure of control over an extended period of time, it is also not surprising that depression, which could be expected to lower both medication and lifestyle adherence, had a significant impact on the ability to reach goal. Further support for the impact of depression on glycemic control is provided by the difference between those with signs of depression but subsequent treatment ( $48 \%$ at goal) with an antidepressant and those with no antidepressant (38\% at goal). These data suggest that depression symptoms make it harder to reach A1c clinical goals but that treatment with an antidepressant may ameliorate this effect.

The results for blood pressure were surprising. Although test rates did not differ by depression symptom presence, the group with symptoms of depression had a significantly higher percentage at the systolic goal. When the group with depression symptoms was examined relative to their subsequent treatment with antidepressants, the treated group had a significantly higher percentage of patients at clinical goals for both systolic (64\%) and diastolic blood pressure (51\%). This is clearly a finding that needs further research.

The lipid results seem to display yet a third pattern. The only clearly significant difference was found in the testing rate. Patients with signs of depression had a lower testing rate $(56 \%$ vs $68 \%)$ than those without signs of depression. This may be due to the higher level of complexity involved in 
preparing for and having a fasting lipid panel. For a patient experiencing symptoms of depression, fasting and travel to a lab may not be as manageable. LDL levels were not different between patients with depression symptoms and those not reporting symptoms.

To the extent that depression interferes with the ability of the patient to reach clinical goals, it increases a patient's risk of future comorbidity. The diabetes patient who has low adherence to their diabetes management, lipid, or blood pressure medication(s) as a result of depression is placed at greater risk for both micro- and macrovascular comorbid events. Diabetes patients are not alone in being negatively impacted by the presence of depression. Studies exist that suggest that both coronary artery disease and hypertension are negatively impacted by the presence of depression. ${ }^{19-21}$

Among the limitations of this study are the reduction in the study population size necessitated by the need to get consent for chart audits and limiting the subjects to those with pharmacy coverage. We also recognize that this approach is limited in that depressed patients who are not being successfully treated at the time of the survey are more likely to report symptoms of depression, whereas patients under successful treatment for depression are less likely to report symptoms of depression. The higher percentage of patients reporting symptoms of depression in our study than found in other studies suggests that the identification method used in this study may be more sensitive than that used in other investigations. Many of the other investigations tried to limit themselves to major depressive disorder (MDD). We were more liberal and included patients reporting symptoms of depression.

The results of this study suggest that depression symptoms among diabetes patients are associated with their ability to reach some clinical goals. However, this impact varies greatly among these goals. Glycohemoglobin control is reduced in diabetes patients even though test rates are high. This may be a result of reduced adherence to medications and lifestyle adjustments. Blood pressure seems to be lower among patients with symptoms of depression and under medication treatment for depression. Depression also seems to make it harder to obtain the test results for lipids. It is simply not enough for the physician to identify depression in their diabetes patients; they need to consider how the depression symptoms and their treatment might impact the pa- tient's ability to reach each of their individual clinical goals.

We thank the many medical groups and their associated Institutional Review Boards that participated in the Quest study.

\section{References}

1. Kessler RC, Berglund P, Demler O, et al. The epidemiology of major depressive disorder: results from the National Comorbidity Survey Replication (NCS-R). JAMA 2003;289:3095-105.

2. Anderson RJ, Freedland KE, Clouse RE, Lustman PJ. The prevalence of comorbid depression in adults with diabetes: a meta-analysis. Diabetes Care 2001; 24:1069-78.

3. Clouse RE, Lustman PJ, Freedland KE, Griffith LS, McGill JB, Carney RM. Depression and coronary heart disease in women with diabetes. Psychosom Med 2003;65:376-83.

4. Rugulies R. Depression as a predictor for coronary heart disease. a review and meta-analysis. Am J Prev Med 2002;23:51-61.

5. de Groot M, Anderson R, Freedland KE, Clouse RE, Lustman PJ. Association of depression and diabetes complications: a meta-analysis. Psychosom Med 2001;63:619-30.

6. de Groot M, Jacobson AM, Samson JA, Welch G. Glycemic control and major depression in patients with type 1 and type 2 diabetes mellitus. J Psychosom Res 1999;46:425-35.

7. Gary TL, Crum RM, Cooper-Patrick L, Ford D, Brancati FL. Depressive symptoms and metabolic control in African-Americans with type 2 diabetes. Diabetes Care 2000;23:23-9.

8. Karlson B, Agardh CD. Burden of illness, metabolic control, and complications in relation to depressive symptoms in IDDM patients. Diabet Med 1997;14: $1066-72$.

9. Lustman PJ, Anderson RJ, Freedland KE, de Groot M, Carney RM, Clouse RE. Depression and poor glycemic control: a meta-analytic review of the literature. Diabetes Care 2000;23:934-42.

10. Lustman PJ, Clouse RE. Depression in diabetic patients: the relationship between mood and glycemic control. J Diabetes Complications 2005;19:113-22.

11. Lustman PJ, Griffith LS, Clouse RE, Cryer PE. Psychiatric illness in diabetes mellitus. Relationship to symptoms and glucose control. J Nerv Ment Dis 1986;174:736-42.

12. Van der Does FE, De Neeling JN, Snoek FJ, et al. Symptoms and well-being in relation to glycemic control in type II diabetes. Diabetes Care 1996;19: 204-10.

13. Katon WJ, Lin EH, Russo J, et al. Cardiac risk factors in patients with diabetes mellitus and major depression. J Gen Intern Med 2004;19:1192-9. 
14. The CDC Diabetes Cost-effectiveness Group. Costeffectiveness of intensive glycemic control, intensified hypertension control, and serum cholesterol level reduction for type 2 diabetes. JAMA 2002;287: 2542-51.

15. U.K. Prospective Diabetes Study Group. U.K. prospective diabetes study 16 . Overview of 6 years' therapy of type II diabetes: a progressive disease. Diabetes $1995 ; 44: 1249-58$.

16. Solberg LI, Engebretson KI, Sperl-Hillen JM, Hroscikoski MC, O'Connor PJ. Are claims data accurate enough to identify patients for performance measures or quality improvement? The case of diabetes, heart disease, and depression. Am J Med Qual 2006; 21:238-45.

17. Corson K, Gerrity MS, Dobscha SK. Screening for depression and suicidality in a VA primary care set- ting: 2 items are better than 1 item. Am J Manag Care 2004;10:839-45.

18. Whooley MA, Avins AL, Miranda J, Browner WS. Case-finding instruments for depression. Two questions are as good as many. J Gen Intern Med 1997; 12:439-45.

19. Kronish IM, Rieckmann N, Halm EA, et al. Persistent depression affects adherence to secondary prevention behaviors after acute coronary syndromes. J Gen Intern Med 2006;21:1178-83.

20. Scalco AZ, Scalco MZ, Azul JB, Lotufo Neto F. Hypertension and depression. Clinics 2005;60:24150.

21. Shimbo D, Davidson KW, Haas DC, Fuster V, Badimon JJ. Negative impact of depression on outcomes in patients with coronary artery disease: mechanisms, treatment considerations, and future directions. J Thromb Haemost 2005;3:897-908. 